\title{
Wie mit Populisten umgehen? Demokratie- und planungstheoretische Perspektiven für Planungspraxis und Planungsforschung
}

\author{
Pia Thiele (), Markus Leibenath (1) \\ Eingegangen: 21. Januar 2021 - Angenommen: 6. Mai 2021 - Online veröffentlicht: 7. Juni 2021
}

\begin{abstract}
Zusammenfassung
Das Erstarken populistischer Kräfte stellt die räumliche Planung vor neue Herausforderungen, weil daraus sowohl inhaltliche als auch verfahrensbezogene Konflikte entstehen können. Ziel des Beitrags ist es, drei demokratie- und planungstheoretisch begründete Perspektiven auf Populismus und Planung zu entwickeln und Schlussfolgerungen für Planungspraxis und Planungsforschung zu ziehen. Dabei handelt es sich um elitenorientierte, rational-technokratische Theorien, um deliberative, beteiligungsorientierte Theorien sowie um radikaldemokratisch-agonistische Theorien. Übersetzt man diese theoretischen Perspektiven in planerische Grundorientierungen, dann ergibt sich ein differenzierter Blick auf fünf mögliche Optionen zum Umgang mit populistischen Akteuren und Tendenzen in der Planungspraxis: ,ignorieren; ,argumentieren', ,integrieren', exkludieren' und ,profitieren: So könnten Planer und Populisten unter bestimmten Annahmen zu Verbündeten werden, die beispielsweise das Interesse an der Re-Politisierung bestehender Zustände teilen. Und schließlich werden aus den verschiedenen theoretischen Blickwinkeln heraus Themen und Fragen für weitergehende Untersuchungen vorgeschlagen.
\end{abstract}

Pia Thiele, Leibniz-Institut für ökologische Raumentwicklung, Weberplatz 1, 01217 Dresden, Deutschland p.thiele@ioer.de

Prof. Dr. Markus Leibenath, Fachgebiet Landschaftsplanung und Kommunikation, Universität Kassel, Gottschalkstraße 26, 34127 Kassel, Deutschland

m.leibenath@uni-kassel.de

Leibniz-Institut für ökologische Raumentwicklung, Weberplatz 1, 01217 Dresden, Deutschland

๔ 2021 Leibenath; licensee oekom verlag. This Open Access article is published under a Creative Commons Attribution 4.0 International License.
Schlüsselwörter: Demokratietheorien - kommunikative Planungstheorie - rationale Planungstheorie - agonistische Planungstheorie - Rollen von Planern

How to deal with populists? Democratic and planning theoretical perspectives in view of planning practice and research

\begin{abstract}
The rise of populist forces presents a new challenge to spatial planning because of its potential for conflict in substantial and procedural terms. The aim of the article is to develop three perspectives on populism and planning based on democracy and planning theory and to draw conclusions for planning practice and planning research. The theories consulted are elitist, rational-technocratic theories, deliberative, participatory theories as well as radical democratic-agonistic theories. If we are to translate theses perspectives into planners' hypothetical courses of actions, a nuanced picture of possible strategies of action in dealing with populist actors and tendencies comes about. Planning practitioners may choose strategies like 'ignoring', 'arguing', 'integrating', 'excluding' and 'profiting from' populists. As such, planners and populists might become allies sharing a common interest in repoliticizing current conditions. Concluding, with said theoretical perspectives in mind, the contribution offers topics and questions for further research.
\end{abstract}

Keywords: Democratic theories - Communicative planning theory - Rational planning theory - Agonistic planning theory - Roles of planners 


\section{Einleitung}

Räumliche Planer ${ }^{1}$ sind in ihrer Arbeit mehr und mehr mit dem Erstarken populistischer Kräfte innerhalb der Gesellschaft konfrontiert - in vielen Teilen der Welt. Dazu zählen in den USA die Tea-Party-Bewegung und große Teile der Republikanischen Partei, die französische Sammlungsbewegung Rassemblement National, die internationale OccupyBewegung, die spanische Podemos-Partei, die italienische Lega-Partei und die Fünf-Sterne-Bewegung sowie die Alternative für Deutschland (AfD), um nur einige Beispiele zu nennen. Manche dieser Gruppierungen sind eher dem rechten und andere eher dem linken Teil des politischen Spektrums zuzuordnen.

In den Politikwissenschaften wird mit unterschiedlichen Populismus-Begriffen gearbeitet. Es besteht jedoch weitgehend Einigkeit darüber, dass populistische Parteien und Bewegungen in der Regel mindestens drei Merkmale aufweisen, und zwar unabhängig von ihrer inhaltlich-ideologischen Orientierung (vgl. Mudde/Rovira Kaltwasser 2019): Erstens suggerieren sie eine Spaltung der jeweiligen Gesellschaft in Volk und Elite (Mudde/Rovira Kaltwasser 2019: 25) und leiten daraus eine Repräsentationslücke zwischen Regierenden und Regierten ab (Jörke/Selk 2017: 67). Darauf aufbauend wenden sie zweitens eine Mobilisierungslogik an, der zufolge „,möglichst breite Bevölkerungssektoren als ,das Volk' gegen eine ,Elite“ mobilisiert werden“" (Marchart 2017: 11). Und drittens behaupten Populisten, die Interessen des vermeintlich wahren Volkes zu vertreten, das als homogen imaginiert wird, woraus sich eine antipluralistische Haltung ergibt (Mudde/Rovira Kaltwasser 2019: 25)In Deutschland sind insbesondere die Stimmengewinne der populistischen AfD auch für die räumliche Planung relevant. Denn AfD-Vertreter sind - entsprechend ihrer Erfolge bei Kommunal- und Landtagswahlen - in planerischen Entscheidungsgremien aktiv. Daraus können sich zum einen inhaltliche Konflikte ergeben. So möchte die AfD etwa die Dekarbonisierung der Wirtschaft im Rahmen der sogenannten Großen Transformation beenden und plädiert gegen einen weiteren Ausbau der Windenergie (Alternative für Deutschland 2017: 65f.). Dies steht in Widerspruch zu zentralen Aufgaben der räumlichen Planung, etwa der Windenergie substanziell Raum zu geben (vgl. Wirth/ Leibenath 2017). Zum anderen können Probleme auf proze$\mathrm{du}$

\footnotetext{
1 Aus stilistischen Gründen wird in diesem Text bei Sammelbezeichnungen für nicht näher spezifizierte Personengruppen durchgängig das generische Maskulinum verwendet. Damit sind jedoch ausdrücklich Menschen jeglicher geschlechtlichen Orientierung gemeint.
}

raler Ebene entstehen, weil zumindest Teile der AfD latent oder offen antidemokratische Positionen vertreten und Verschwörungsmythen verbreiten (Kailitz 2020). Außerdem stellen sie - wie etwa im Bezug auf den Klimawandel gut gesicherte wissenschaftliche Erkenntnisse in Frage. Dies läuft dem Ansatz der räumlichen Planung in Deutschland zuwider, Pläne auf der Grundlage wissenschaftlicher Erkenntnisse zu erstellen sowie unterschiedliche Interessen in demokratischen Abwägungs- und Entscheidungsprozessen auszubalancieren (vgl. Leibenath 2019). Daher stehen räumliche Planer in Deutschland vor der praktischen Frage, wie sie mit den Vertretern einer populistischen Partei wie der AfD umgehen sollen.

Populistische Phänomene haben auch unter planungstheoretischen Gesichtspunkten in den letzten Jahren wachsende Beachtung erfahren. Beispielsweise appelliert Sager (2020) an kritische Planungsforscher, neben Neoliberalismus auch autoritären Populismus verstärkt in den Blick $\mathrm{zu}$ nehmen, und zeigt auf, welche Herausforderungen sich daraus insbesondere im Hinblick auf kommunikative Planungstheorien ergeben. Ebenfalls dezidiert planungstheoretisch argumentieren Griggs und Howarth (2008) in ihrer Fallstudie über den Widerstand gegen den Ausbau des Flughafens London-Stansted. Unter Rückgriff auf die poststrukturalistische Populismus-Theorie von Laclau (2007) zeichnen sie nach, wie lokale Protestanliegen mit einer allgemeineren populistischen Rhetorik und entsprechenden Veränderungsstrategien verbunden werden können. Andere Autoren analysieren die Einflussnahme populistischer Akteure auf Planungsprozesse. So beschreibt Filion (2011, 2018), wie sich Torontos Stadtplanungspolitik durch die Wahl eines rechtspopulistischen Bürgermeisters verändert hat, nachdem er das Budget für den öffentlichen Nahverkehr gekürzt und den motorisierten Personenverkehr aufgewertet hat. Weitere Studien widmen sich der Tea Party und arbeiten heraus, wie deren Repräsentanten versuchen, die Deutungshoheit über Planungsprozesse zu erlangen, während sie gleichzeitig räumliche Planung im Allgemeinen (Trapenberg Frick 2013) sowie Klimaanpassungspläne im Speziellen ablehnen (Foss/Howard 2015). Ferner gibt es Untersuchungen, die sich zwar auf Populismus im Zusammenhang räumlicher Konflikte beziehen, allerdings ohne dabei planerische Aspekte eingehender zu thematisieren. Dazu gehören etwa Arbeiten im Kontext der Energiewende (Eichenauer/Reusswig/Meyer-Ohlendorf et al. 2018; Selk/ Kemmerzell/Radtke 2019; Reusswig/Lass/Bock 2020) oder eine Fallstudie von Kurtenbach (2019) über die Ausgrenzung von Geflüchteten.

Hinsichtlich des Stands der Forschung zu Populismus im Kontext räumlicher Fragen ergibt sich somit ein heterogenes Bild. Es besteht aus einzelnen Untersuchungen, die teilweise auf bestimmte Planungs- und Populismustheorien Bezug nehmen, teilweise aber auch rein empirisch au 
Tabelle 1 Überblick über die ausgewählten Demokratie- und Planungstheorien

\begin{tabular}{llll}
\hline Demokratietheorien & $\begin{array}{l}\text { Elitenorientierte } \\
\text { Demokratietheorie }\end{array}$ & $\begin{array}{l}\text { Deliberative, beteiligungsorientierte } \\
\text { Demokratietheorie }\end{array}$ & $\begin{array}{l}\text { Radikale } \\
\text { Demokratietheorie }\end{array}$ \\
$\begin{array}{l}\text { Korrespondierende } \\
\text { Planungstheorien }\end{array}$ & $\begin{array}{l}\text { Rationale, technokratische } \\
\text { Planungstheorie }\end{array}$ & Kommunikative Planungstheorie & $\begin{array}{l}\text { Agonistische } \\
\text { Planungstheorie }\end{array}$ \\
\hline
\end{tabular}

sgerichtet sind oder aber keinen Planungsbezug aufweisen. Außerdem wird das Verhältnis von Planung zu Populismus - je nachdem, welcher theoretische Zugang gewählt wird ganz unterschiedlich bewertet, zum Beispiel kritisch im Falle von Sager (2020) oder eher neutral im Falle von Griggs und Howarth (2008).

Anknüpfend an diese Beobachtungen ist es Ziel dieses Beitrags, erstens mehrere kontrastierende demokratie- und planungstheoretisch begründete Perspektiven auf das Phänomen ,Populismus und Planung ' zu skizzieren. Dabei bilden Demokratietheorien gewissermaßen das Bindeglied der Systematisierung, weil sowohl Populismus- als auch Planungstheorien eng mit demokratietheoretischen Prämissen zusammenhängen. Diese Art von Theorievergleich ist einerseits unter planungswissenschaftlichen Gesichtspunkten relevant: Indem wir aufzeigen, welche Art von Forschungsfragen sich aus den verschiedenen Perspektiven ergeben, kann unsere Gegenüberstellung dazu beitragen, die theoretische Fundierung zukünftiger empirischer Untersuchungen $\mathrm{zu}$ verbessern. Und andererseits stellen die theoretischen Überlegungen Reflexionsangebote für die tägliche Planungspraxis dar, die sich mit populistischen Herausforderungen konfrontiert sieht. Das zweite Ziel des Beitrags besteht daher darin zu zeigen, welche Implikationen sich aus den verschiedenen theoretischen Perspektiven für Planungspraxis und Planungsforschung ableiten lassen.

Im Folgenden wird zunächst ein Überblick darüber gegeben, welche demokratie- und planungstheoretischen Perspektiven wir vergleichen und welche Vergleichsparameter wir heranziehen (Kapitel 2). In den anschließenden drei Kapiteln erörtern wir die verschiedenen Ansätze anhand dieser Parameter. Im letzten Teil setzen wir die Perspektiven zueinander in Beziehung (Kapitel 6) und ziehen Schlussfolgerungen für Planungspraxis und Planungsforschung (Kapitel 7).

\section{Die gewählten Theorien und die Vorgehensweise}

Sowohl Demokratie- als auch Planungstheorien sind Gegenstand vielfältiger Systematisierungen (vgl. z. B. Hillier/ Healey 2010; Lembcke/Ritzi/Schaal 2012; Allmendinger 2017; Schmidt 2019). In diesem Beitrag konzentrieren wir uns auf drei demokratietheoretische Stränge, die das zeitgenössische Denken über Demokratie nachhaltig ge prägt haben und sich stark voneinander unterscheiden. Außerdem weisen sie enge Bezüge zu verschiedenen Planungstheorien und Planungsverständnissen auf und bieten jeweils spezifische Zugänge zu populistischen Phänomenen. Bei den drei Strängen handelt es sich um elitenorientierte, deliberative und radikale Demokratietheorien, die wir nachfolgend kurz vorstellen (vgl. Tabelle 1).

Die elitenorientierte Spielart der Demokratietheorie wird prominent von Joseph Schumpeter vertreten, der sich dabei unter anderem auf Max Weber bezieht. Demnach ist Demokratie ein durch Wahl hervorgebrachtes repräsentatives Herrschaftssystem, in dem den demokratisch gewählten Politikern ein weitreichender Entscheidungsspielraum zukommt (Schumpeter 2018 [1942]: 386). Giovanni Sartori, der auf Schumpeters Ideen aufbaut und eine moderne liberale Elitentheorie entwickelte, spricht von Demokratie als „selektive[m] System konkurrierender gewählter Minderheiten [...], eine[r] selektive[n] Polyarchie“" (Sartori 1997: 177). Diese elitentheoretischen Gedanken weisen Gemeinsamkeiten mit den frühen planungstheoretischen Überlegungen von Autoren wie Andreas Faludi (1973) zu rationaler Planung auf. Das „Rationalitätsideal“ (Wiechmann 2019: 5), das darin zutage tritt, ist auch für die heutige Planungspraxis noch von Bedeutung.

Deliberative, beteiligungsorientierte Demokratietheoretiker, als deren einflussreichster Vertreter Jürgen Habermas gilt, postulieren, dass politische Fragen idealerweise durch öffentliche „Prozeduren der Beratung und Beschlussfassung“ (Habermas 1992: 359) in machtfreien Räumen getroffen werden sollten, um einen gesellschaftlich akzeptierten Konsens herzustellen (Buchstein 2011: 322). Diese Ideen bilden den Ausgangspunkt vieler kommunikativer, partizipativer Planungstheorien (Healey 1992b; Healey 1997; Forester 1999; Forester 2009).

Eine der bekanntesten Varianten radikaler Demokratietheorie wurde von Ernesto Laclau und Chantal Mouffe (2014) zunächst gemeinsam formuliert und in den letzten Jahren von Mouffe $(2007,2014)$ zum Konzept der „agonistischen Demokratie“ weiterentwickelt. Für sie ist Demokratie der Kampf um Hegemonie zwischen politischen Gegnern und somit der Kampf um die Repräsentation bisher ausgeschlossener Gruppen (Mouffe 2014: 184). Daran anschließend diskutieren wir radikale Demokratie- und Planungstheoretiker (Metzger 2018; Legacy/ Metzger/Steele et al. 2019) anhand des Begriffs der Post 
politik (Crouch 2008; Mouffe 2011; Blühdorn 2013) Defizite gegenwärtiger (liberaler) Demokratien und Planungssysteme, aber auch Möglichkeiten, diese neu zu beleben.

Bei der Beschreibung und beim Vergleich dieser drei demokratietheoretischen Zugänge orientieren wir uns an mehreren Parametern. Dabei handelt es sich um das der jeweiligen Theorie zugrunde liegende Verständnis von Demokratie, von Bürgern und Partizipation, von Konflikt, von Macht und schließlich von Legitimation. Diese grundlegenden politikwissenschaftlichen Kategorien (vgl. Comtesse/FlügelMartinsen/Martinsen et al. 2019; Schmidt 2019: 514ff.) werden im Hinblick auf Planungstheorien um die Fragen ergänzt, welche Art von Problemen den Gegenstand von Planung bilden, worin Ziel und Wesen der Planung bestehen, mit welchen Technologien - im Sinne von Ansätzen und Verfahren - in der Planung typischerweise gearbeitet und wie die Rolle von Planern gesehen wird.

\section{Elitenorientierte Demokratietheorie und rationale, technokratische Planungstheorie}

Die Entwicklung repräsentativer, liberaler Demokratien ging während der Nachkriegszeit in vielen westlichen Staaten Hand in Hand mit dem Aufbau rationalistischer Planungssysteme. Das Attribut, elitenorientiert` mag in diesem Zusammenhang irritieren; aber wie wir im Folgenden zeigen, bezeichnet es eine wesentliche Gemeinsamkeit zwischen der entsprechenden Demokratietheorie und technokratischen, rationalistischen Planungsverständnissen. Genau an dieser Elitenorientierung setzte ab den 1980erJahren Kritik an, die zur Formulierung stärker deliberativ, kommunikativ und partizipativ ausgerichteter Demokratieund Planungstheorien führte (vgl. Kapitel 4). Planung ist stets untrennbar mit politischen Entscheidungsverfahren verwoben, weswegen Kühn (2020: 3) den Terminus ,politics of planning" verwendet und Planung in all ihren Phasen als politischen Prozess betrachtet. Gleichzeitig müssen Pläne die geltenden Gesetze berücksichtigen, auf einer soliden, aktuellen Datengrundlage beruhen und mit anerkannten Verfahren gemäß dem Stand der Technik erstellt werden. Daher bildet das Modell rationaler Planung, auch wenn es Züge eines „Mythos“ trägt, nicht ganz zu Unrecht seit Langem den ,eigentliche[n] Kern des Selbstverständnisses von Planern“ (Wiechmann 2019: 4).

Elitenorientierte Demokratietheoretiker stellen sich Demokratie als politisches System vor, in dem der gewählten Volksvertretung und der Verwaltung ein großes Maß an Verantwortung und Entscheidungsspielraum zugesprochen wird. Giovanni Sartori spricht in diesem Zusammenhang davon, dass „Demokratie [...] ein selektives System ko nkurrierender gewählter Minderheiten“ oder „eine selektive Polyarchie“" (Sartori 1997: 177) sein solle. Damit meint er eine Herrschaft der ausgewählten Vielen, die in ihrer jeweiligen Einflusssphäre das politische Geschehen lenken.

Bürgern kommt in diesem System lediglich die Aufgabe $\mathrm{zu}$, sich an den regelmäßig stattfindenden Wahlen zu beteiligen (Sartori 1997: 94). Weitergehende Beteiligungsformen werden abgelehnt, weil es dem ,typischen Bürger“ an politischem Sachverstand fehle: Der falle ,auf eine tiefere Stufe der gedanklichen Leistung, sobald er das politische Gebiet betritt" (Schumpeter 2018 [1942]: 355). Bürger stünden der Politik generell fern (vgl. Schaal 2012: 455), was mangelndes Urteilsvermögen und Verantwortungsgefühl zur Folge habe (Schumpeter 2018 [1942]: 354). Deswegen wird dem Fachwissen in Bürokratie und Politik und der Kompetenz große Bedeutung beigemessen, aber wenig Wert auf Bürgerbeteiligung gelegt.

In diesem Zuge ist es kaum überraschend, dass politische Konflikte vor allem im Hinblick auf die Führungsebene thematisiert werden: Streitfragen würden für das Volk, nicht durch das Volk entschieden (Schumpeter 2018 [1942]: 358). Über die demokratischen Werte sollte nach Ansicht der Elitentheoretiker ein von möglichst vielen getragener Grundkonsens bestehen, denn andernfalls gerate die Demokratie in Gefahr (Schumpeter 2018 [1942]: 400). Insofern sind elitentheoretische Demokratiemodelle im Kern konsensorientiert. Konkrete politische Streitfragen sollen allein von Politikern durch Mehrheitsbeschlüsse entschieden werden.

In Anlehnung an Max Webers Definition von Macht als „Chance, innerhalb einer sozialen Beziehung den eigenen Willen auch gegen Widerstreben durchzusetzen," (Weber 1990: 28) geht es hier vor allem um Macht über andere (,power over“, Partzsch 2017: 200), also darum, sich in Konkurrenzsituationen gegenüber anderen zu behaupten (Schumpeter 2018 [1942]: 384; Schmidt 2019: 176).

Elitentheoretische Demokratiemodelle gehen davon aus, dass sich die Legitimation politischer Entscheidungen in erster Linie aus deren Ergebnissen ergibt („Output-Legitimation"). Über den Mechanismus des Wettbewerbs ließen sich die besten Politiker rekrutieren und durch den demokratischen Rechtsstaat könne die Fairness des Verfahrens sichergestellt werden (Schaal 2012: 452). Die von diesen „erlesenen Besten“ gefassten politischen Beschlüsse zeichneten sich dadurch aus, dass sie von der bürgerlichen Mehrheit befürwortet werden würden (Schaal 2012: 445).

Indem Elitentheorien die Relevanz von Fach- und Führungspersonal betonen, beschränkte Beteiligungsformen für Bürger vorsehen sowie Problemlösungsorientierung und Output-Legitimation hervorheben, weisen sie Gemeinsamkeiten mit rationalen Planungstheorien auf. Die Art von Problemen, auf die Planung gemäß diesem Planungsverständnis reagieren soll, sind primär administr 
ativer und technischer Natur, beispielsweise überbordende Kosten, Funktionsschwächen technisch-praktischer Art oder Informationsdefizite (Faludi 1973: 106f.). Das übergeordnete Ziel von Planung besteht demnach darin, technisch und ökonomisch optimale Lösungen zu finden (Faludi 1973: 51; 75f.; Brand/Gaffikin 2007: 307; Wiechmann 2019: 5). Daher stellen faktenbasierte und rationale Abwägungen in Form von Kosten-Nutzen-Analysen, Modellierungen sowie „Versuch und Irrtum“ (Faludi 1973: 108 ff.) zentrale Technologien oder Instrumente rationaler Planung dar.

Ähnlich der Vorstellung von Elitentheoretikern, der zufolge die Bürger lediglich das Führungspersonal wählen, nehmen diese in rationalen Planungstheorien allenfalls die Rolle von Informanten ein, die Kontextinformationen zur Verfügung stellen, darüber hinaus aber nicht an Planungsprozessen beteiligt werden (Faludi 1973: 120). Im Gegensatz dazu sind Planer wissenschaftlich geschulte Fachleute, die über Fakten- und Methodenwissen verfügen (Allmendinger 2017: 70). Solchen Planerpersönlichkeiten ,steht die Politik im Weg“" (Forester 1982: 69). Denn für Politik sind Aushandlungsprozesse und Entscheidungsfindungen zwischen verschiedenen Akteuren üblich und notwendig. Für rationale Planung hingegen sind Aushandlungen entbehrlich, da sich optimale Lösungen allein durch Anwendung geeigneter fachlich-technischer Methoden ermitteln lassen (Forester 1982: 69).

Was folgt daraus für den Umgang mit populistischen Phänomenen? Innerhalb dieser theoretischen Perspektive stehen Planer in einem potenziell ambivalenten Verhältnis zu populistischen Akteuren: Sie können sie als Störfaktor zurückweisen oder sich - in Anbetracht der von beiden Gruppen geteilten antipolitischen Grundhaltung - mit ihnen verbünden. Denn einerseits stellen Populisten, die demokratische Spielregeln missachten oder gar außer Kraft setzen wollen, aus elitenorientierter Perspektive eine Gefahr dar: Der Verführung des Volkes durch antipluralistische Leitgedanken müsse eine Wählerschaft und ein Parlament mit genügend hohem intellektuellem und moralischem Niveau gegenüberstehen, um die Demokratie zu verteidigen (Schumpeter 2018 [1942]: 398). So gesehen werden populistische Anliegen als störend und demokratiegefährdend wahrgenommen. Und umgekehrt können die Vertreter einer problemorientierten, rationalen und gesetzeskonformen Planung leicht zum Angriffsziel von Populisten werden.

Andererseits könnte es jedoch auch zum Schulterschluss zwischen Populisten und rationalistisch orientierten Planern kommen, weil beide eigentümlich apolitisch sind (Bickerton/Invernizzi Accetti 2017: 326f.). Während Populisten Anspruch erheben zu wissen, was das, wahre' Gemeinwohl sei, neigen Experten aufgrund ihrer fachlichen Ausbildung leicht dazu, für sich zu beanspruchen, ,bessere Entscheidungen treffen zu können. Populisten und Tec hnokraten können gleichermaßen schlecht mit Meinungsvielfalt und Opposition umgehen. Tendenziell betrachten sie ihre Gegner wahlweise als „Feinde des Volkes“ oder als „Feinde der Wahrheit“ (Moore/Invernizzi-Accetti/ Markovits et al. 2020: 735). Populismus und Technokratie sind somit ,ihrer inneren Logik nach antipluralistisch“. Sie sind nicht auf eine demokratische Diskussionskultur angewiesen oder verachten diese gar, ,denn die richtige Antwort steht ja ohnehin bereits fest“ (Müller 2016: 70).

\section{Deliberative, beteiligungsorientierte Demokratietheorien und kommunikative Planungstheorie}

In den letzten Jahrzehnten konnte man eine geradezu explosionsartige Verbreitung neuartiger Beteiligungs- und Kooperationsformate in der räumlichen Planung beobachten: Leitbildprozesse, Planungszellen, Landschaftswerkstätten, kooperative Stadtentwicklung und Governance lauten einige der entsprechenden Stichworte. Auch in weiten Bereichen der formalen Planung gehört Bürger- und Öffentlichkeitsbeteiligung mittlerweile zum Standard und ist gesetzlich verankert. Damit haben Gesetzgeber und Planungsgremien auf die Mitwirkungsansprüche der Bürger reagiert, die seit der „partizipatorischen Revolution“ (Blühdorn 2013: 152) der 1960er- und 1970er-Jahre kontinuierlich gestiegen sind. Diese planungspraktischen Entwicklungen korrespondieren mit deliberativen, beteiligungsorientierten Demokratie- und Planungstheorien, auf die wir im Folgenden eingehen.

Die Idee der Verständigung durch rationalen und herrschaftsfreien Diskurs bildet die Grundlage deliberativer Demokratietheorien. Habermas (1992: 365) spricht davon, dass sich Demokratie durch ,ein[e] dezentriert[e] Gesellschaft" auszeichnet, ,die allerdings mit der politischen Öffentlichkeit eine Arena für die Wahrnehmung, Identifizierung und Behandlung gesamtgesellschaftlicher Probleme" hervorbringt. Deliberation ist eine Prozedur der Beratschlagung, die öffentlich sowie frei von Macht und Gewalt, zwischen gleichberechtigten Teilnehmenden, offen für alle Probleme und Fragestellungen und unter Berücksichtigung potenziell aller gesellschaftlichen Gruppen, Perspektiven und Argumente verläuft (Landwehr 2012: 362). Das bedeutet, dass alle Bürger politische Prozesse und Diskussionen mitgestalten dürfen und sollen, oder anders gesagt: Jeder darf und soll mitreden. Damit ist Demokratie im deliberativen Modell tendenziell basisdemokratisch angelegt.

Bürger werden als kommunikativ kompetent und als lernbereit eingeschätzt. Ihre politischen Überzeugungen gelten als aufklärungsbedürftig und -fähig. Das wirksamste Mittel zum Austausch von Positionen stellt die politis 
che Kommunikation der Bürger untereinander dar (Buchstein 2011: 321). Weiterhin wird angenommen, dass sie gemeinwohlorientiert handeln (Landwehr 2012: 360f.). Diese Annahme beruht auf der Idee des ,zwanglosen Zwangs des besseren Arguments“ (Habermas 1992: 370). Damit wird die Vermutung ausgedrückt, dass sich in deliberativen Kommunikationsprozessen solche Argumente durchsetzen werden, die dem Gemeinwohl und nicht Partikularinteressen dienen. Streng genommen gehen deliberative über rein beteiligungsorientierte Demokratietheorien hinaus: Sie setzen breite Beteiligung voraus, aber verlagern den Fokus von der Öffnung auf die Organisation von Entscheidungsprozessen (Blühdorn 2013: 85).

Konflikte, gegensätzliche Anliegen und widerstreitende Überzeugungen bilden den Ausgangspunkt von Deliberationsprozessen. Laut Habermas müssen Konflikte in modernen, komplexen Gesellschaften kommunikativ bewältigt werden. Die kooperative und gewaltfreie Regelung des $\mathrm{Zu}$ sammenlebens stelle einerseits „Solidarität unter Fremden“ her und gewährleiste andererseits das Recht, ,füreinander Fremde zu bleiben" (Habermas 1992: 374). Im Laufe der Verständigung sollen gemeinsame Interessen durch den Austausch von Argumenten gefunden werden, damit eine von allen Beteiligten getragene Lösung möglich wird (Habermas 1992: 386). Ziel von Deliberation ist daher die Herstellung von Konsens.

Mit der Kategorie ,Macht" verbinden deliberative Demokratietheoretiker sowohl negative als auch positive Aspekte. Einerseits gilt Macht im Weber'schen Sinne als verzerrender Einfluss, den es zugunsten einer weitreichenden und inklusiv angelegten Verständigung zwischen den Beteiligten zu minimieren gilt. Andererseits ist Macht in Anlehnung an Hannah Arendt auch die Fähigkeit, gemeinsam zu handeln (Habermas 1992: 183 f.; Iser/Strecker 2010: 118f.). Durch die Deliberation wird kommunikative Macht erzeugt, die insofern produktiv ist, als dass sie gemeinschaftliches Handeln ermöglicht und dazu beiträgt, neue Wirklichkeiten zu erschaffen (,,power with“/,,power to“, Partzsch 2017: 200).

Legitimität wird durch den Deliberationsprozess als solchen erzeugt, wie Habermas (1992: 367) unterstreicht: „Die deliberative Politik gewinnt ihre legitimierende Kraft aus der diskursiven Struktur einer Meinungs- und Willensbildung, die ihre sozialintegrative Funktion nur dank der Erwartung einer vernünftigen Qualität ihrer Ergebnisse erfüllen kann." Das bedeutet, dass Deliberation zwar hauptsächlich input- und throughput-orientiert, aber auch auf einen entsprechenden Output angewiesen ist. Die Bedeutung von Beteiligung liegt auf der Hand (input). Darüber hinaus muss das Verfahren den deliberativ-demokratischen Ansprüchen entsprechen (throughput); und schließlich legitimiert sich der Prozess auch über Ergebnisse, die im Idealfall von allen Beteiligten anerkannt werden (output).
Das planungswissenschaftliche Pendant deliberativer Demokratietheorien ist in kommunikativen Planungstheorien zu finden. Ausgangspunkt kommunikativer Planung ist die Feststellung, dass rationale Planungsansätze oftmals nur schwach normativ fundiert sind (Allmendinger 2017: 241) und soziale Ungerechtigkeit als Nebenprodukt von rationaler Planung entstehen kann (Brand/Gaffikin 2007: 288; Zimmermann 2019: 18). Im Kern adressieren kommunikative Planungstheoretiker verschiedene Formen von Gerechtigkeit. Einerseits geht es ihnen um Verfahrensgerechtigkeit, also darum, im Vorfeld Regeln für die Teilnahme an kommunikativen Planungsprozessen festzulegen, sodass sich die Teilnehmer idealerweise im Ergebnis auf eine Lösung einigen können (Sager 2018: 93). Andererseits steht Verteilungsgerechtigkeit im Fokus, die insbesondere für die Tätigkeit von Planern relevant ist, da diese eine Vermittlerrolle zwischen verschiedenen Interessen einnehmen (Healey 2003: 104; Allmendinger 2017: 264). Weiterhin spielt das Konzept der „Gerechtigkeit als Anerkennung“ (Honneth 2004) eine wichtige Rolle, weil es auch darum geht, diverse Perspektiven anzuerkennen und die Anliegen marginalisierter Gruppen einzubeziehen (Innes/Booher 2015: $201 \mathrm{ff}$.). Kommunikative Planungstheoretiker lassen sich aber auch von der Annahme leiten, dass Deliberation und das Bewusstsein für Gerechtigkeitsprobleme zu sachlich-materiell besseren Lösungen führen, weil lokales Wissen in den Planungsprozess einfließt und kollektive Lernprozesse begünstigt werden (Innes/Booher 2016: 9).

Planung sollte dementsprechend allen Stimmen Gehör verschaffen und unterschiedliche Perspektiven zur Geltung bringen, um bedarfsorientiertere Planungsergebnisse zu erzielen (Forester 2012: 24). Genau wie von den Vertretern deliberativer Demokratietheorie vorgezeichnet, soll dies durch argumentativen Austausch unter möglichst herrschaftsfreien und konsensorientierten Bedingungen ablaufen (Sager 2018: 93). Alle Beteiligten sollten gleichberechtigt sein. Bürger sollten mit Entscheidern auf Augenhöhe kommunizieren können (Brand/Gaffikin 2007: 292). Planer fungieren als Moderatoren, die den Austausch zwischen allen Beteiligten initiieren und fördern (Forester 1982: 73; Innes/Booher 2016: 10; Allmendinger 2017: 256).

Aus der Sicht kommunikativer Planungstheorien werden Planer durch Populisten in einen Zwiespalt gebracht. Denn einerseits ähneln sich Populismus und kommunikative Planung in dem anti-elitären Anspruch, mit, gewöhnlichen Leuten' ins Gespräch zu kommen: „Both bodies of ideas realize that elites can manipulate and co-opt procedural democracy, and both favour some control over globalized market forces" (Sager 2020: 94). In einem kommunikativen Planungsprozess wäre es also denkbar, dass Populisten zunächst - wie alle anderen Beteiligten - als legitime Teilnehmer des deliberativen Verfahrens anerkannt werden. D 
as würde bedeuten, dass kommunikativ orientierte Planer Populisten wie allen anderen Beteiligten das Recht einräumen mitzureden.

Andererseits ist es vorstellbar und sogar wahrscheinlich, dass populistische Akteure von kommunikativ eingestellten Planern als störend wahrgenommen werden, sobald sie deliberative, pluralistische Aushandlungsverfahren obstruieren (Sager 2020: 20). Deliberative Demokratie beruht auf temporären, wiederkehrenden Aushandlungen des Gemeinwohls - egal ob in repräsentativen Institutionen oder der breiten Öffentlichkeit. Demgegenüber geben Populisten vor zu wissen, was das Gemeinwohl ist. Deliberation ist für sie redundant, ebenso wie die Partizipation von Bürgern (Abts/Rummens 2007: 417). Daher stellt der Umgang mit Populisten für Planer im Rahmen deliberativer, kommunikativer Theorien eine Gratwanderung zwischen TeilnehmenLassen und Ausschluss dar.

\section{Radikale Demokratietheorie und agonistische Planungstheorie}

Dieses Bündel von Theorien bekommt zunehmende Beachtung, weil sich angesichts der Erfahrungen mit deliberativen, partizipativen Ansätzen in Teilen von Planungspraxis und -forschung Ernüchterung eingestellt hat. Denn in vielen Fällen ziehen deliberative Beteiligungsformate in der Praxis vor allem Privilegierte an und marginalisieren die ohnehin bereits benachteiligten Gruppen tendenziell weiter. Somit werden sie dem eigenen Anspruch eines inklusiven Diskurses oftmals nicht gerecht (Sack 2019: $671 \mathrm{ff}$.; Krüger 2020: 20f.). Außerdem sehen sich Planer etwa in Zusammenhang mit Projekten wie Stuttgart 21 oder dem Bau von Windkraftanlagen immer häufiger mit tiefgreifenden Konflikten konfrontiert, bei denen deliberative, konsensorientierte Verfahren an ihre Grenzen stoßen. Hier setzen radikale, agonistische Demokratie- und Planungstheorien an. Sie betrachten Konflikte als Normalfall der Demokratie oder gar als demokratiefördernd (Hillier 2003; Kühn 2020). Außerdem richten sie ihr Augenmerk auf Strategien zum Umgang mit neoliberalen und antidemokratischen Tendenzen in der Stadtund Regionalplanung (Purcell 2009).

Radikale Demokratietheoretiker gehen davon aus, dass Gesellschaften von vielfältigen Antagonismen durchzogen sind und dass kollektiven, demokratischen Entscheidungsprozessen per se etwas Konflikthaftes zu eigen ist. Dieses Konflikthafte wird ,das Politische“ genannt (Mouffe 2007: 15). Hauptaufgabe der Demokratie ist es, das Politische in die ,richtigen' Bahnen zu leiten, indem politische Feindschaft (Antagonismus) in reflektierte, sachorientierte Gegnerschaft (Agonismus) überführt wird. Opponenten sollen sich nicht unbedingt einigen, sondern ihr Gegenüber und dessen konträre Standpunkte aner kennen (Mouffe 2007: 29f.). Auf dieser Basis können politische Auseinandersetzungen stattfinden, in deren Rahmen die Opponenten versuchen, durch Koalitionsbildung Hegemonie zu erlangen, das heißt, die Oberhand über den politischen Prozess zu gewinnen (Mouffe 2007: 27).

Radikale Demokratietheoretiker begreifen die Kategorie des ,Bürgers“ als eine Form der politischen Identität, die sich den politischen Prinzipen der pluralistischen Demokratie, also der Geltendmachung von Freiheit und Gleichheit - im Sinne gleicher Rechte und Freiheit von Unterdrückung - für alle verschreibt (Mouffe 1992: 30). Damit unterscheiden sie sich sowohl von liberalen Konzeptionen, die ,den Bürger' als weitgehend passiven Träger und Empfänger bestimmter Rechte betrachten, als auch von kommunitaristischen Vorstellungen des Bürgers als Teil einer Gemeinschaft mit geteilten Werten (und somit der Gleichheit gegenüber der Freiheit den Vorzug geben) (Mouffe 1992: $29 \mathrm{f}$.). In einem radikaldemokratischen Verständnis zeichnen sich Bürger dadurch aus, dass sie sich identifizieren mit den Anliegen emanzipatorischer Bewegungen, die sich für die Rechte von Frauen, Arbeitern oder Menschen mit anderer Hautfarbe sowie gegen Umweltzerstörungen einsetzen. Ein solcherart aktivistisch verstandenes Eintreten für Gleichheit und Freiheit durchzieht die Handlungen radikaldemokratischer Bürger in allen Lebensbereichen. Daraus lässt sich ableiten, dass nach radikaldemokratischen Prinzipien handelnde Akteure politische Aktivisten sind, die agonistische Kämpfe austragen und sich stets gegen Beherrschung und Unterdrückung einsetzen (Mouffe 1992: $31 \mathrm{f}$.).

In Situationen, in denen sowohl die Problemdefinitionen als auch die anzustrebenden Gemeinwohlziele umstritten sind, kann sich Politik weder über ihren demokratischen Input - also die Beteiligung und Befragung der Mitglieder des Gemeinwesens - noch über ihren inhaltlich-materiellen Output legitimieren. Stattdessen ist radikaldemokratische Politik in dem Maße legitimiert, wie es ihr gelingt, Konflikte mithilfe geeigneter Verfahren und Mechanismen zu ,zähmen ' und die einander gegenüberstehenden Parteien dazu zu bringen, die Rechtmäßigkeit der Gegner und ihrer Standpunkte anzuerkennen (Mouffe 2007: 29f.). Das bedeutet, dass wir es hier in erster Linie mit verfahrensbezogener (Throughput-)Legitimation zu tun haben.

Gleichzeitig ist es radikalen Demokratietheoretikern wichtig, dass Konflikte und grundlegende ideologische Differenzen nicht unterdrückt werden. Daher kritisieren sie sogenannte postpolitische Tendenzen, die Swyngedouw (2011: 266) folgendermaßen definiert: „Post-politics reduces the political terrain to the sphere of consensual governing and policy-making, centred on the technical, managerial and consensual administration (policing) of environmental, social, economic or other domains [...]." Wenn das bestehende soziale und ökono 
mische Gefüge nicht grundsätzlich diskutiert wird oder werden kann, dann können auch Wahlen als Inszenierung oder Simulation (Mouffe 2011: 4) wahrgenommen werden und den Boden für populistische Argumentationen bereiten. Postpolitische Tendenzen manifestieren sich nach Blühdorn (2013: 157) etwa in der Entpolitisierung zuvor politisierter Bürger, Themen und Institutionen, im zunehmenden Einfluss von Lobbygruppen, Expertenkommissionen und Gerichten sowie darin, dass ökonomische Effizienz zur allumfassenden Referenznorm erhoben wird mithin also in Entwicklungen, die oft auch mit dem Attribut ,neoliberal ' versehen werden. Unter dem Strich steht Postpolitik also dem zentralen Anliegen radikaldemokratischer Demokratietheoretiker entgegen, ,,eine agonistische Auseinandersetzung zwischen unterschiedlichen gesellschaftlichen Projekten“ (Mouffe 2018: 28) zu ermöglichen.

Der Kategorie ,Macht" wird in radikaldemokratischen Ansätzen ein großer Stellenwert beigemessen. Ausgehend von einem poststrukturalistischen Begriff von Diskurs als artikulatorischer Praxis, die soziale Beziehungen konstituiert und organisiert (Howarth 2010: 311; Leibenath/Otto 2012: $121 \mathrm{ff}$.), wird genau diese artikulatorische Praxis als machtförmig konzeptualisiert (Kühn 2020: 9). Diskurse bringen einerseits geteilte Vorstellungen des gesellschaftlichen Miteinanders hervor. Andererseits gründen Diskurse auch auf machtvollen Ausschließungen. Die dadurch unterdrückten Möglichkeiten können allerdings jederzeit reaktiviert werden, weswegen Diskurse niemals vollständig geschlossen, sondern stets unvollständig und brüchig sind (Mouffe 2007: 27). In diesem Spannungsverhältnis zwischen geteilten und ausgeschlossenen Vorstellungen des Gesellschaftlichen findet das Ringen um Hegemonie statt. Hegemonie ist die Etablierung einer Ordnung, die sich durch bestimmte Vorstellungen des Gemeinwohls auszeichnet und die aus dem Bestreben resultiert, möglichst viele konträre politische Positionen entweder zu integrieren und dadurch den eigenen Diskurs zu verbreitern oder aber diese konträren Positionen auszuschließen und zu marginalisieren (Maeße/Nonhoff 2014: 386 ff.; Kühn 2020: 9). In diesem Sinne ist Hegemonie der Kampf um gesellschaftliche Vorherrschaft und verweist darauf, wie Macht soziale Beziehungen prägt. Macht ist demnach in erster Linie struktureller Natur und wirkt stets sowohl produktiv und ermöglichend (,power to“) als auch repressiv und ausschließend (,power over“) (Partzsch 2017: 200). Demnach muss es in der radikaldemokratischen (Planungs)praxis darum gehen, strukturelle, diskursive Machteffekte in ihrer Kontingenz offenzulegen und dadurch Spielräume für die Repräsentation bisher ausgeschlossener Gruppen zu eröffnen (Mouffe 2014: 184).

Agonistische Planungstheorien beziehen sich in ihrer Problembeschreibung auf die von radikalen Demokratietheoretiken beschriebenen postpolitischen Zuständ
(McClymont 2019: 15). Demnach gerieten Beteiligungsangebote unter postpolitischen Bedingungen im Extremfall $\mathrm{zu}$ einer Fassade, hinter der sich ein unanfechtbarer Wachstumsimperativ verstecke (Metzger 2018: 185). Eine Diskussion über Alternativen falle umso schwerer, je mehr das Politische verdrängt werde (Eraydın/Tasan-Kok 2019: 29 f.). Aus diesem Grund schlagen agonistische Planungstheoretiker vor, Planung explizit als politischen Prozess $\mathrm{zu}$ verstehen und anzulegen (Hillier 2003: 54; Oosterlynck/Swyngedouw 2010: 1582). Darüber hinaus solle eine agonistisch informierte Planung radikale Alternativen zu wachstumsorientierten Visionen entwickeln oder wenigstens zulassen, dass diese von anderen Akteuren eingebracht werden (Purcell 2009: 160). Da Konsens kein vorrangiges Ziel agonistischer Planung ist, geht es eher darum, Fragen zu stellen mit dem Ziel, den Status quo in Zweifel zu ziehen (Hillier 2003: 42). Brüche und Widersprüche sollen mithilfe von unkonventionellen Methoden - zum Beispiel durch Kunstprojekte - inszeniert oder aufgedeckt werden. Planer können dabei die Rolle von politischen Aktivisten oder Fragestellern einnehmen (,professional idiots“, Bylund/ Byerley 2014: 147).

In der radikalen Demokratietheorie sind unterschiedliche Sichtweisen auf populistische Phänomene $\mathrm{zu}$ finden (Agridopoulos/Kim 2019: 593, 601), aber Populismus wird keineswegs generell negativ gesehen. So formuliert etwa Laclau (2007) ein sozialtheoretisches Populismuskonzept, das Populismus als Normalfall in Demokratien begreift. Demnach müsse jedes politische Projekt populistische Züge aufweisen, um erfolgreich sein zu können. Laclau beschreibt eine allgemeine populistische Handlungslogik, die er für jegliche hegemonialen politischen Projekte als charakteristisch betrachtet. Sie besteht daraus, eine politische Einheit - ,das Volk' - zu konstruieren und gegen ein antagonistisches Anderes zu stellen, um somit den eigenen politischen Forderungen Gewicht zu verleihen. Ganz in diesem Sinne erklärt Mouffe (2018: 21), Populismus sei ,eine Art, Politik zu betreiben“, „die zu verschiedenen Zeiten und an verschiedenen Orten unterschiedliche Formen annehmen kann und mit einer Vielzahl institutioneller Rahmenbedingungen kompatibel ist“". Die Ursache für das Erstarken von Rechtspopulismus liegt für Mouffe im postpolitischen Charakter der liberalen Demokratie. Diese biete den Menschen keine tatsächlichen Alternativen, weswegen rechtspopulistische Slogans attraktiv würden (Mouffe 2007: 94; Mouffe 2018: 26f.). Daran zeigt sich, dass aus agonistischer Perspektive nicht Populismus, sondern postpolitische Zustände das eigentliche Problem darstellen. Gemäß agonistischer Theorien stehen Planer vor der Herausforderung, ein als zu de-politisiert (oder: zu technokratisch) empfundenes System zu re-politisieren. V 
Tabelle 2 Vergleich der ausgewählten drei theoretischen Perspektiven

\begin{tabular}{|c|c|c|c|}
\hline \multirow{2}{*}{$\begin{array}{l}\text { Vorherrschendes } \\
\text { Verständnis ... }\end{array}$} & \multicolumn{3}{|c|}{... innerhalb der theoretischen Perspektiven: } \\
\hline & $\begin{array}{l}\text { Elitenorientierte Demokra- } \\
\text { tietheorie in Verbindung mit } \\
\text { rationaler, technokratischer } \\
\text { Planungstheorie }\end{array}$ & $\begin{array}{l}\text { Deliberative, beteiligungsorientierte } \\
\text { Demokratietheorie in Verbindung mit } \\
\text { kommunikativer Planungstheorie }\end{array}$ & $\begin{array}{l}\text { Radikale Demokratietheorie in Verbin- } \\
\text { dung mit agonistischer Planungstheo- } \\
\text { rie }\end{array}$ \\
\hline ... von Demokratie & $\begin{array}{l}\text { Herrschaft der ausgewählten } \\
\text { Vielen }\end{array}$ & $\begin{array}{l}\text { Deliberative, partizipative Entschei- } \\
\text { dungsfindung }\end{array}$ & 'Zähmung' gesellschaftlicher Konflikte \\
\hline $\begin{array}{l}\text {... von ,Bürger' und } \\
\text { Partizipation }\end{array}$ & $\begin{array}{l}\text { Weitgehend passive Bürger; } \\
\text { sehr eingeschränkte Beteili- } \\
\text { gungsmöglichkeiten }\end{array}$ & $\begin{array}{l}\text { Kompetente und aktive Bürger, } \\
\text { deren Beteiligung erwünscht und } \\
\text { notwendig ist }\end{array}$ & $\begin{array}{l}\text { Politische Aktivisten; Beteiligung in } \\
\text { politischen Kämpfen }\end{array}$ \\
\hline ... von Konflikten & $\begin{array}{l}\text { Werden innerhalb der Eliten } \\
\text { ausgetragen }\end{array}$ & $\begin{array}{l}\text { Ausgangszustand, den es in einen Kon- } \\
\text { sens zu überführen gilt }\end{array}$ & $\begin{array}{l}\text { Unabdingbares Phänomen, das allen- } \\
\text { falls temporär eingehegt werden kann }\end{array}$ \\
\hline ... von Macht & $\begin{array}{l}\text { Personengebundene Ressour- } \\
\text { ce und als Quelle von Repres- } \\
\text { sion (power over) }\end{array}$ & $\begin{array}{l}\text { Personengebundene Ressource: Quelle } \\
\text { von Repression (power over), aber auch } \\
\text { Voraussetzung kollektiven Handelns } \\
\text { (power with/power to) }\end{array}$ & $\begin{array}{l}\text { Weitgehend entpersonalisierte, struk- } \\
\text { turelle Macht, die sowohl repressiv } \\
\text { (power over) als auch produktiv (power } \\
\text { to) wirken kann }\end{array}$ \\
\hline $\begin{array}{l}\text {... der Art, wie po- } \\
\text { litisches Handeln } \\
\text { legitimiert wird }\end{array}$ & Output-orientiert & $\begin{array}{l}\text { Vor allem input- und throughput-, aber } \\
\text { auch output-orientiert }\end{array}$ & Throughput-orientiert \\
\hline $\begin{array}{l}\text {... der Probleme, } \\
\text { auf die räumliche } \\
\text { Planung reagieren } \\
\text { sollte }\end{array}$ & $\begin{array}{l}\text { Administrativ-technische Pro- } \\
\text { blemstellungen }\end{array}$ & $\begin{array}{l}\text { Ungerechtigkeit in unterschiedlichen } \\
\text { Manifestationen }\end{array}$ & $\begin{array}{l}\text { Postpolitische Zustände; neoliberaler } \\
\text { Status quo }\end{array}$ \\
\hline $\begin{array}{l}\text {... von Wesen und } \\
\text { Ziel von Planung }\end{array}$ & $\begin{array}{l}\text { Ermittlung fachlich-metho- } \\
\text { disch und technisch optimaler } \\
\text { Lösungen }\end{array}$ & $\begin{array}{l}\text { Inklusive Beteiligungsprozesse und ziel- } \\
\text { gruppenorientierte Lösungen }\end{array}$ & Re-Politisierung; Bürger aktivieren \\
\hline $\begin{array}{l}\text {... von Technologien } \\
\text { der Planung }\end{array}$ & $\begin{array}{l}\text { Faktenbasierte, rationale } \mathrm{Ab} \text { - } \\
\text { wägung }\end{array}$ & Deliberative Verfahren & $\begin{array}{l}\text { Kultivierung des politischen Streits; } \\
\text { unkonventionelle Ansätze (Provokatio- } \\
\text { nen) }\end{array}$ \\
\hline $\begin{array}{l}\text {... der Rolle von } \\
\text { Planern }\end{array}$ & $\begin{array}{l}\text { Wissenschaftlich geschulte } \\
\text { Fachleute }\end{array}$ & Moderatoren & $\begin{array}{l}\text { Fragesteller; politisierende Provokateu- } \\
\text { re }\end{array}$ \\
\hline $\begin{array}{l}\text {... populistischer } \\
\text { Phänomene }\end{array}$ & $\begin{array}{l}\text { Störfaktor } \\
\text { oder } \\
\text { gleichgerichtete, weil ebenfalls } \\
\text { antipluralistische Kraft }\end{array}$ & $\begin{array}{l}\text { Störfaktor } \\
\text { oder } \\
\text { eine Stimme unter vielen, denen zuge- } \\
\text { hört werden sollte }\end{array}$ & $\begin{array}{l}\text { Normalfall der Demokratie; begrüßens- } \\
\text { werte Beiträge zur (Re-)Politisierung } \\
\text { der Gesellschaft }\end{array}$ \\
\hline
\end{tabular}

on dieser Warte aus betrachtet sind populistische Strategien begrüßenswert, insoweit sie eine solche Re-Politisierung begünstigen.

\section{Vergleichende Diskussion}

In diesem Beitrag haben wir zwar eine Minimaldefinition von Populismus als einem Typus elitenkritischer und antipluralistischer politischer Strömungen zugrunde gelegt, aber ansonsten versucht, uns jeder eigenen Bewertung populistischer Phänomene zu enthalten. Ziel war es, Vorschläge zu unterbreiten, wie das Verhältnis von Populismus und räumlicher Planung aus unterschiedlichen demokratie- und planungstheoretischen Perspektiven erforscht werden kann und welche Implikationen sich aus diesen verschiedenen Zugängen für die Planungspraxis ergeben. Dazu wurden elitenorientierte, deliberativ-beteiligungsorienti erte und radikale Demokratietheorien sowie damit korrespondierende Planungstheorien ausgewählt, die wir anhand eines Sets von Kriterien miteinander verglichen haben (vgl. die

Übersicht in Tabelle 2).

Welche sind die zentralen Unterschiede und Gemeinsamkeiten zwischen den betrachteten Theorien - insbesondere in Hinblick auf die uns in erster Linie interessierenden Dimensionen ,Planung' und ,Populismus“? In elitenorientierten Demokratie- und den korrespondierenden rationalen Planungstheorien wird davon ausgegangen, dass räumliche Planung vor allem mit administrativ-technischen Problemen konfrontiert ist, die auf fachlich-methodischem Weg durch evidenzbasierte Abwägung gelöst werden können und sollen. Ganz anders dagegen ist die Sicht deliberativer, kommunikativer Theorien: Hier beschäftigt sich räumliche Planung in erster Linie mit Gerechtigkeitsproblemen, die dadurch zu bearbe 
Wie mit Populisten umgehen? Demokratie- und planungstheoretische Perspektiven für Planungspraxis und ...

Tabelle 3 Optionen zum Umgang mit populistischen Akteuren und Tendenzen in Abhängigkeit von der planerischen Grundorientierung

\begin{tabular}{|c|c|c|c|}
\hline \multirow{2}{*}{$\begin{array}{l}\text { Optionen zum Umgang mit populistischen } \\
\text { Akteuren und Tendenzen }\end{array}$} & \multicolumn{3}{|c|}{ Planerische Grundorientierungen } \\
\hline & Technokratisch & Kommunikativ & Radikaldemokratisch \\
\hline Ignorieren & + & - & $(+)$ \\
\hline Argumentieren & $(+)$ & + & + \\
\hline Integrieren & - & + & + \\
\hline Exkludieren & - & + & $(+)$ \\
\hline Profitieren & + & - & + \\
\hline
\end{tabular}

$\left({ }^{+}{ }^{“}=\right.$ Option steht zur Verfügung,,$(+)^{\prime}=$ Option steht eventuell zur Verfügung, ,-“ = Option steht nicht zur Verfügung)

iten sind, dass alle relevanten Gruppen gehört und beteiligt werden und dass zielgruppenorientierte, konsensuale Lösungen gefunden werden. Radikale Demokratietheorien und die damit verbundenen agonistischen Planungstheorien wiederum betonen die positive Bedeutung von Konflikten. Folglich sollte räumliche Planung dazu beitragen, latente Konflikte offenzulegen - etwa durch gezielte Provokationen - und so einer politischen Debatte zuzuführen. Dabei wird unterstellt, dass sich Konflikte in der Regel nicht lösen, sondern bestenfalls einhegen und zähmen lassen.

Ähnlich unterschiedlich sind die Rollen, die den Bürgern und den Planern zugeschrieben werden. Aus elitentheoretischer Sicht sollten Bürger zwar politische Informationen, aber nur minimale Mitbestimmungsmöglichkeiten in Form von Wahlen erhalten. Planer werden als wissenschaftlich geschulte Fachleute gesehen, denen aufgrund ihrer Expertise eine hohe Entscheidungskompetenz zukommt. Bei den Kommunikations- und Beteiligungsprozessen, die im Fokus deliberativer Theorien stehen, begegnen sich die Bürger und die Planer sowie die Bürger untereinander auf Augenhöhe. Planer können dabei die Moderation oder (fachlich-inhaltlich sowie organisatorisch) die Prozessbegleitung übernehmen. Und von der Warte radikaldemokratischer, agonistischer Theorien aus betrachtet sollten Bürger wie Planer idealerweise in die Rolle politischer Aktivisten schlüpfen, die sich offensiv mit den unvermeidlichen Konflikten und gesellschaftlichen Bruchlinien auseinandersetzen, allerdings auf möglichst zivilisierte Weise. Dabei geht es darum, Planungsprozesse zu re-politisieren, die durch die Verengung politischer Diskussions- und Handlungsspielräume zuvor ent-politisiert worden sind.

Weil sie wissenschaftlich gestützte Planungsverfahren und pluralistische Abwägungsprozesse gleichermaßen ablehnen, können populistische Akteure als Störfaktoren bewertet werden, und zwar sowohl aus der Sicht der elitenorientierten als auch der deliberativen, kommunikativen Theorien. Aus deliberativ-kommunikativer Perspektive ist ebenfalls eine neutrale Bewertung von Populismus als legitimer Stimme, der wie anderen Stimmen zugehört werden muss, möglich. Ein anderer Forschungsstrang verweist auf die antidemokratischen Gemeinsamkeiten vo n Technokratie und Populismus, die in der Praxis durchaus einen Zusammenschluss dieser Akteure bedeuten könnte. Tendenziell positiv werden populistische Phänomene aus der Perspektive radikaldemokratischer, agonistischer Theorien beurteilt, weil sie dazu beitragen können, die bestehenden gesellschaftlichen Verhältnisse grundlegend zu hinterfragen und postpolitische Zustände aufzubrechen - allerdings nur solange, wie sich keine Konflikte mit dem radikaldemokratischen Eintreten für Freiheit und Gleichheit abzeichnen.

\section{Schlussfolgerungen für Planungspraxis und Planungsforschung}

Wie kann in der Planungspraxis mit populistischen Akteuren und Tendenzen umgegangen werden? Erste Anhaltspunkte dazu haben wir bereits jeweils am Ende der Kapitel 3, 4 und 5 sowie in der letzten Zeile von Tabelle 2 gegeben. Die dort benannten theoretisch hergeleiteten Sichtweisen und Einschätzungen - ,Störfaktor', ,gleichgerichtete Kraft', ,eine Stimme unter vielen ' und ,Normalfall der Demokratie - lassen sich unter planungspraktischen Gesichtspunkten weiter differenzieren und akzentuieren. Unseres Erachtens ergeben sich daraus im Wesentlichen fünf Optionen zum Umgang mit populistischen Akteuren und Tendenzen, die wir mit ,ignorieren', ,argumentieren', ,integrieren', ,exkludieren' und ,profitieren' überschrieben haben (vgl. Tabelle 3). Des Weiteren gehen wir davon aus, dass sich die drei von uns gewählten demokratieund planungstheoretischen Perspektiven in idealtypische planerische Grundorientierungen übersetzen lassen. „Idealtypisch", weil es sich um holzschnittartige Abstraktionen handelt, die nicht mit realen Personen gleichzusetzen sind. Welche Optionen zur Verfügung stehen, hängt davon ab, von welcher planerischer Grundorientierung man ausgeht (vgl. Tabelle 2). Inwieweit konkrete Planerpersönlichkeiten ihre Grundeinstellungen ändern können sowie ob eine Person - je nach Situation - unterschiedlichen Rationalitäten folgen kann, wird seit Langem in der planungstheoreti 
schen Forschung diskutiert (Healey 1992a; Hellmich/ Lamker/Lange 2017) und soll an dieser Stelle ausgeklammert bleiben.

Die erste Option - ,ignorieren “ - läuft darauf hinaus, dass in den Planungsbehörden einfach keine Notiz genommen wird von populistischen Bestrebungen. Kommunikativ orientierten Planern steht diese Option nicht zur Verfügung, weil sie den Anspruch haben, jeden Akteur und jede gesellschaftliche Gruppe gleichermaßen zu Wort kommen zu lassen. Technokratisch eingestellte Akteure können diese Option jedoch verfolgen, indem sie populistische VorstöBe als offensichtlich unbegründet, weil unwissenschaftlich zurückweisen. Ähnlich könnten auch radikaldemokratisch orientierte Planer verfahren, wobei andere Optionen für sie naheliegender sein dürften.

Die Option ,argumentieren' bedeutet, dass sich Planer mit Populisten auseinandersetzen - sei es unter inhaltlichfachlichen oder unter verfahrensbezogenen Gesichtspunkten. Weil Populisten demokratisch-pluralistischen Entscheidungsprozessen tendenziell skeptisch gegenüberstehen, dürften sich gerade kommunikativ orientierte Planer gezwungen sehen, mit Populisten über prozessuale Fragen zu argumentieren. Technokratisch eingestellte Planer würden, wenn überhaupt, eher über inhaltliche Aspekte wie etwa die Qualität der verwendeten wissenschaftlichen Daten diskutieren. Weil radikaldemokratische Planer eine positive Einstellung zu Konflikten haben, würden sie sich bereitwillig in inhaltliche oder auch verfahrensbezogene Wortgefechte mit Populisten stürzen.

,Integrieren' und ,exkludieren' sind zwei Optionen, die sich auf die Mitwirkung von Nicht-Experten - also ,normalen Bürgern ' - an planerischen Entscheidungsverfahren beziehen. Weil Bürgerbeteiligung, die über das bloße Informieren hinausgeht, im Weltbild technokratisch-elitär orientierter Planer keinen Platz hat, sind diese beiden Optionen für sie von vornherein irrelevant. Für kommunikativ orientierte Planer ist es selbstverständlich, gemäß ihrem Ideal der möglichst breiten Beteiligung auch Populisten in den Diskussions- und Deliberationsprozess einzubeziehen. Dies gilt allerdings nur so lange, wie Populisten nicht das Mitspracherecht anderer in Frage stellen oder sich den Prinzipien eines herrschaftsfreien Diskurses widersetzen. In dem Maße, in dem Populisten eben diese Regeln missachten, würden sich kommunikative Planer gezwungen sehen, Populisten auszuschließen und als illegitime Sprecher zu brandmarken. Dies gilt weitgehend auch für radikaldemokratisch orientierte Planer, wobei hier die Option, exkludieren ' nur als allerletztes Mittel in Frage käme, etwa dann, wenn Populisten rassistische oder andere menschenverachtende Positionen verträten.

Mit der Option ,profitieren ' ist gemeint, dass sich Planer die Ansätze von Populisten zunutze machen. So könnten technokratisch orientierte Planer den Schulterschlu ss mit Populisten suchen, die Parlamentarismus und Pluralismus eher distanziert gegenüberstehen. Ebenso können radikaldemokratische Planer in Populisten Verbündete sehen, weil sie ein gemeinsames Interesse daran haben, den gesellschaftlichen Status quo grundlegend zu hinterfragen. Für kommunikativ orientierte Planer gibt es hingegen hinsichtlich populistischer Tendenzen und Kräfte nichts zu gewinnen. Sie müssen stets fürchten, dass faire, rationale und gemeinwohlorientierte Entscheidungsverfahren von Populisten sabotiert werden.

Wie könnten die von uns dargelegten demokratie- und planungstheoretischen Perspektiven für empirische Untersuchungen genutzt werden? Aus elitentheoretischer Perspektive läge das Hauptaugenmerk weniger auf prozessualen oder politischen, sondern vor allem auf physisch-materiellen und ökonomischen Aspekten. Planungsforscher könnten beispielsweise danach fragen, inwieweit die Einwendungen oder sonstigen Äußerungen von Planungsbeteiligten auf wissenschaftlichen Grundlagen beruhen. Dementsprechend würden die Positionen populistischer Akteure im Sinne eines Faktenchecks auf Wahrheitsgehalt und Schlüssigkeit überprüft. Außerdem wäre zu klären, ob die Standpunkte im Einklang stehen mit den jeweils relevanten rechtlichen und sachlich-fachlichen Normen. Gemäß der technokratischen Logik wären populistische Forderungen im Kontext der räumlichen Planung anhand von Kriterien wie Schlüssigkeit, Zweckmäßigkeit und Effizienz zu prüfen. Eine solche Analyse würde nicht automatisch auf eine Entkräftung oder Abwertung populistischer Standpunkte hinauslaufen. Denkbar sind auch Konstellationen, in denen eine Popularisierung von Forderungen zu beobachten ist, die sich am aktuellen Stand der Forschung orientieren, also beispielsweise ein wissenschaftlich fundierter Klimaschutz-Populismus.

Aus der Perspektive deliberativer, kommunikativer Theorien würde sich der Untersuchungsfokus auf Kommunikations- und Entscheidungsprozesse verlagern. Zunächst wäre zu fragen, wie diese Prozesse im konkreten Fall organisiert und inszeniert werden. In aller Regel gibt es hier große Spielräume, beispielsweise weil Planung frühzeitige freiwillige Beteiligungsformate anbieten könnte. Außerdem könnten unterschiedliche Zielgruppen adressiert und mit einem breiten Spektrum von Kommunikationsstilen und -kanälen angesprochen werden. Darüber hinaus könnten Argumentationsmuster betrachtet werden, und zwar auf Seiten aller Planungsbeteiligten. Hierbei wären insbesondere die sprachlichen Interaktionen interessant, also mit welchen Argumenten Planer auf Populisten reagieren und umgekehrt. Außerdem könnte analysiert werden, inwiefern vermeintliche Populisten tatsächlich populistisch argumentieren und ob es bestimmte Themen gibt, die besonders häufig als Ausgangspunkt populistischer Argumentationen dienen. Relevant sind auch Aspekte, die unt 
er dem Schlagwort ,Kommunikationskultur ‘ subsumiert werden können: Wie gehen die Beteiligten miteinander um? Ist die Kommunikation von gegenseitiger Anerkennung und von Respekt geprägt, was ja gerade bei deliberativen Ansätzen so essenziell ist? Und schließlich müsste beleuchtet werden, in welchem Maße es den Beteiligten gelingt, konsensuale Lösungen zu finden.

Planungsforscher, die sich aus radikaldemokratischer, agonistischer Perspektive mit Populismus auseinandersetzen wollen, könnten zunächst analysieren, welche politischen Forderungen der betreffende populistische Diskurs beinhaltet und inwiefern es sich dabei um ein potenziell hegemoniales Projekt handelt. Von einem potenziell hegemonialen Projekt wäre dann zu sprechen, wenn etwa energieund klimapolitische Positionen mit zahlreichen weiteren Forderungen verknüpft würden, etwa solchen nach gleichwertigeren und gerechteren Lebensverhältnissen, einem besseren Schutz der Gesundheit und der Schaffung neuer Arbeitsplätze. Außerdem müsste untersucht werden, wie radikal die populistischen Forderungen sind und inwieweit sie sich mithin eignen, einen postpolitischen Status quo in Frage zu stellen und zur Re-Politisierung von Diskussionen und Planungsprozessen beizutragen. $\mathrm{Zu}$ fragen wäre auch, ob die beteiligten Planer eine solche Re-Politisierung als Chance oder Bedrohung begreifen. Und schließlich wäre es interessant zu erfahren, inwieweit sich Verlauf und Ergebnisse von Planungsprozessen unter dem Einfluss von Populisten ändern, also ob eine eventuelle Re-Politisierung dazu führt, dass räumliche Probleme grundsätzlicher und offener diskutiert werden und dass eine größere Vielfalt von Alternativen berücksichtigt wird.

Vor dem Hintergrund der laufenden gesellschaftlichen Umbrüche und der noch notwendigen sozialökologischen Transformationen ist davon auszugehen, dass sich raumbezogene Konflikte in den nächsten Jahren deutlich verschärfen werden. Damit einhergehend könnten sowohl rechts- als auch linkspopulistische Phänomene weiter an Bedeutung gewinnen, und zwar sowohl auf Seiten derer, die am Status quo festhalten möchten (z. B. in Teilen der Gewerkschaftsund Arbeiterbewegung, die vor allem am Erhalt von Arbeitsplätzen und Arbeitnehmerrechten interessiert sind), als auch auf Seiten der Befürworter gesellschaftlicher Veränderungen in Richtung größerer Nachhaltigkeit (z. B. Gruppierungen wie Extinction Rebellion). Daher dürfte es für Planungspraxis und Planungsforschung zukünftig noch dringlicher werden, sich mit dem Verhältnis zwischen Populismus und räumlicher Planung auseinanderzusetzen.

Förderhinweis Der Beitrag ist im Rahmen des Projekts „Wie Planer(innen) gemacht werden: Subjektivierungen von Planer(inne)n im Kontext von Regionalplanung und Windenergie in Deutschland" entstanden, das von der Deutschen Forschungsgemeinschaft (DFG) unter dem Geschäftszeichen LE 2255/6-1 (Projektnummer 401342127) gefördert wird.

\section{Literatur}

Abts, K.; Rummens, S. (2007): Populism versus Democracy. In: Political Studies 55, 2, 405-424. https://doi.org/ 10.1111/j.1467-9248.2007.00657.x

Agridopoulos, A.; Kim, S. (2019): Populismus. In: Comtesse, D.; Flügel-Martinsen, O.; Martinsen, F.; Nonhoff, M. (Hrsg.): Radikale Demokratietheorie. Ein Handbuch. Berlin, 593-603.

Allmendinger, P. (2017): Planning Theory. London.

Alternative für Deutschland (2017): Programm für Deutschland. Berlin.

Bickerton, C.; Invernizzi Accetti, C. (2017): Populism and Technocracy. In: Rovira Kaltwasser, C.; Taggart, P.; Ochoa Espejo, P.; Ostiguy, P. (Hrsg.): The Oxford Handbook of Populism. Oxford, 326-341. https://doi.org/10. 1093/oxfordhb/9780198803560.001.0001

Blühdorn, I. (2013): Simulative Demokratie. Neue Politik nach der postdemokratischen Wende. Berlin.

Brand, R.; Gaffikin, F. (2007): Collaborative planning in an uncollaborative world. In: Planning Theory 6, 3, 282-313. https://doi.org/10.1177/1473095207082036

Buchstein, H. (2011): Jürgen Habermas. In: Massing, P.; Breit, G.; Buchstein, H. (Hrsg.): Demokratietheorien. Von der Antike bis zur Gegenwart. Bonn, 316-322.

Bylund, J.; Byerley, A. (2014): Hopeless postpolitics, professional idiots, and the fate of public space in Stockholm parklife. In: Metzger, J.; Allmendinger, P.; Oosterlynck, S. (Hrsg.): Planning Against the Political. Democratic Deficits in European Territorial Governance. New York, 129-151.

Comtesse, D.; Flügel-Martinsen, O.; Martinsen, F.; Nonhoff, M. (Hrsg.) (2019): Radikale Demokratietheorie. Ein Handbuch. Berlin.

Crouch, C. (2008): Postdemokratie. Frankfurt am Main.

Eichenauer, E.; Reusswig, F.; Meyer-Ohlendorf, L.; Lass, W. (2018): Bürgerinitiativen gegen Windkraftanlagen und der Aufschwung rechtspopulistischer Bewegungen. In: Kühne, O.; Weber, F. (Hrsg.): Bausteine der Energiewende. Wiesbaden, 633-651. https://doi.org/10.1007/ 978-3-658-19509-0_32

Eraydın, A.; Tasan-Kok, T. (2019): Crisis in planning theory. In: Eraydın, A.; Frey, K. (Hrsg.): Politics and Conflict in Governance and Planning. New York, 21-37.

Faludi, A. (1973): Planning Theory. Oxford.

Filion, P. (2011): Toronto's tea party: Right-wing populism and planning agendas. In: Planning Theory and Practice 12, 3, 464-469. https://doi.org/10.1080/14649357.2011. 617504

Filion, P. (2018): Enduring features of the North American suburb: built form, automobile orientation, suburban cul 
ture and political mobilization. In: Urban Planning 3, 4. https://doi.org/10.17645/up.v3i4.1684

Forester, J. (1982): Planning in the face of power. In: Journal of the American Planning Association 48, 1, 67-80. https://doi.org/10.1080/01944368208976167

Forester, J. (1999): The Deliberative Practitioner. Encouraging participatory planning processes. Cambridge.

Forester, J. (2009): Dealing with Differences. Dramas of Mediating Public Disputes. Oxford.

Forester, J. (2012): Learning to Improve Practice: Lessons from Practice Stories and Practitioners' own Discourse Analyses (or Why Only the Loons Show Up). In: Planning Theory and Practice 13, 1, 11-26. https://doi.org/ 10.1080/14649357.2012.649905

Foss, A. W.; Howard, J. (2015): The other end of the spectrum: municipal climate change mitigation planning in the politically conservative Dallas-Fort Worth region. In: Environment and Planning C 33, 6, 1412-1431. https:// doi.org/10.1177/0263774X15614454

Griggs, S.; Howarth, D. (2008): Populism, localism and environmental politics: the logic and rhetoric of the Stop Stansted Expansion campaign. In: Planning Theory 7, 2, 123-144. https://doi.org/10.1177/1473095208090431

Habermas, J. (1992): Faktizität und Geltung. Beiträge zur Diskurstheorie des Rechts und des demokratischen Rechtsstaats. Frankfurt am Main.

Healey, P. (1992a): A planner's day. Knowledge and action in communicative practice. In: Journal of the American Planning Association 58, 1, 9-20. https://doi.org/10. 1080/01944369208975531

Healey, P. (1992b): Planning through debate: The communicative turn in planning theory. In: Town Planning Review 63, 2, 233-249. https://doi.org/10.3828/tpr.63.2. $422 \times 602303814821$

Healey, P. (1997): Collaborative Planning. Shaping Places in Fragmented Societies. Vancouver.

Healey, P. (2003): Collaborative planning in perspective. In: Planning Theory 2, 2, 101-123. https://doi.org/10.1177/ 14730952030022002

Hellmich, M.; Lamker, C. W.; Lange, L. (2017): Planungstheorie und Planungswissenschaft im Praxistest: Arbeitsalltag und Perspektiven von Regionalplanern in Deutschland. In: Raumforschung und Raumordnung | Spatial Research and Planning 75, 1, 7-17. https://doi.org/10.1007/ s13147-016-0464- $x$

Hillier, J. (2003): 'Agon'izing over consensus: Why Habermasian ideals cannot be 'real'. In: Planning Theory 2, 1, 37-59. https://doi.org/10.1177/1473095203002001005

Hillier, J.; Healey, P. (Hrsg.) (2010): The Ashgate Research Companion to Planning Theory. Farnham.

Honneth, A. (2004): Recognition and justice. Outline of a plural theory of justice. In: Acta Sociologica 47, 4, 351-364. https://doi.org/10.1177/0001699304048668

Howarth, D. (2010): Power, discourse, and policy: articulating a hegemony approach to critical policy studies. In: Critical Policy Studies 3, 3/4, 309-335. https://doi.org/ 10.1080/19460171003619725

Innes, J. E.; Booher, D. E. (2015): A turning point for planning theory? Overcoming dividing discourses. In: Planning Theory 14, 2, 195-213. https://doi.org/10. $1177 / 1473095213519356$

Innes, J. E.; Booher, D. E. (2016): Collaborative rationality as a strategy for working with wicked problems. In: Landscape and Urban Planning 154, 8-10. https://doi. org/10.1016/j.landurbplan.2016.03.016

Iser, M.; Strecker, D. (2010): Jürgen Habermas zur Einführung. Hamburg.

Jörke, D.; Selk, V. (2017): Theorien des Populismus zur Einführung. Hamburg.

Kailitz, S. (2020): Sachsen - eine Hochburg der AfD? Entwicklung, Perspektiven und Einordnung der Bundespartei und des sächsischen Landesverbandes. In: Backes, U.; Kailitz, S. (Hrsg.): Sachsen - Eine Hochburg des Rechtsextremismus? Göttingen, 155-200. = Schriften des Hannah-Arendt-Instituts für Totalitarismusforschung 66.

Krüger, T. (2020): Gemeinwohlkonflikte in der Energiewende. Eine radikaldemokratische Perspektive auf Energiekonflikte und die Grenzen der Deliberation. Berlin. = WZB Discussion Paper, SP III 2020-602.

Kühn, M. (2020): Agonistic planning theory revisited: The planner's role in dealing with conflict. In: Planning Theory. https://doi.org/10.1177/1473095220953201

Kurtenbach, S. (2019): Kein Platz für Flüchtlinge? Eine empirische Untersuchung der Ausgrenzung Geflüchteter am Beispiel der Stadt Bautzen. In: Raumforschung und Raumordnung | Spatial Research and Planning 77, 4, 367-382. https://doi.org/10.2478/rara-2019-0029

Laclau, E. (2007): On populist reason. London.

Laclau, E.; Mouffe, C. (2014): Hegemony and socialist strategy. Towards A Radical Democratic Politics. London.

Landwehr, C. (2012): Demokratische Legitimation durch rationale Kommunikation. In: Lembcke, O.; Ritzi, C.; Schaal, G. S. (Hrsg.): Zeitgenössische Demokratietheorie. Band 1: Normative Demokratietheorien. Wiesbaden, 355-385. https://doi.org/10.1007/978-3-531-94161-5_ 12

Legacy, C.; Metzger, J.; Steele, W.; Gualini, E. (2019): Beyond the post-political: Exploring the relational and situated dynamics of consensus and conflict in planning. In: Planning Theory 18, 3, 273-281. https://doi.org/10. $1177 / 1473095219845628$

Leibenath, M. (2019): Berufliche Identitäten von Regionalplanern im Kontext der Windenergienutzung: eine $\mathrm{p}$ 
oststrukturalistische Perspektive. In: Raumforschung und Raumordnung | Spatial Research and Planning 77, 2, 165-180. https://doi.org/10.2478/rara-2019-0008.

Leibenath, M.; Otto, A. (2012): Diskursive Konstituierung von Kulturlandschaft am Beispiel politischer Windenergiediskurse in Deutschland. In: Raumforschung und Raumordnung 70, 2, 119-131. https://doi.org/10.1007/ s13147-012-0148-0

Lembcke, O.; Ritzi, C.; Schaal, G. S. (Hrsg.) (2012): Zeitgenössische Demokratietheorie. Band 1: Normative Demokratietheorien. Wiesbaden. https://doi.org/10.1007/9783-531-94161-5

Maeße, J.; Nonhoff, M. (2014): Macht und Hegemonie im Diskurs. In: Angermüller, J.; Nonhoff, M.; Herschinger, E.; Macgilchrist, F.; Reisigl, M.; Wedl, J.; Wrana, D.; Ziem, A. (Hrsg.): Diskursforschung. Ein interdisziplinäres Handbuch. Band 1: Theorien, Methodologien und Kontroversen. Bielefeld, 386-410.

Marchart, O. (2017): Liberaler Antipopulismus. Ein Ausdruck von Postpolitik. In: Aus Politik und Zeitgeschichte 44/45, 11-16.

McClymont, K. (2019): Articulating virtue: Planning ethics within and beyond post politics. In: Planning Theory 18, 3, 282-299. https://doi.org/10.1177/1473095218773119

Metzger, J. (2018): Postpolitics and planning. In: Gunder, M.; Madanipour, A.; Watson, V. (Hrsg.): The Routledge Handbook of Planning Theory. New York, 180-193.

Moore, A.; Invernizzi Accetti, C.; Markovits, E.; Pamuk, Z.; Rosenfeld, S. (2020): Beyond populism and technocracy: The challenges and limits of democratic epistemology. In: Contemporary Political Theory 90, 4, 730-752. https://doi.org/10.1057/s41296-020-00398-1

Mouffe, C. (1992): Citizenship and Political Identity. In: October 61, 28-32. https://doi.org/10.2307/778782

Mouffe, C. (2007): Über das Politische. Wider die kosmopolitische Illusion. Frankfurt am Main.

Mouffe, C. (2011): ,Postdemokratie‘ und die zunehmende Entpolitisierung. In: Aus Politik und Zeitgeschichte 1/2, 3-5.

Mouffe, C. (2014): Agonistik. Die Welt politisch denken. Berlin.

Mouffe, C. (2018): Für einen linken Populismus. Bonn.

Mudde, C.; Rovira Kaltwasser, C. (2019): Populismus. Eine sehr kurze Einführung. Bonn.

Müller, J.-W. (2016): Schatten der Repräsentation: Der Aufstieg des Populismus. In: Blätter für deutsche und internationale Politik, 4, 63-74.

Oosterlynck, S.; Swyngedouw, E. (2010): Noise reduction: The postpolitical quandary of night flights at Brussels Airport. In: Environment and Planning A 42, 7, 1577-1594. https://doi.org/10.1068/a42269
Partzsch, L. (2017): 'Power with' and 'power to' in environmental politics and the transition to sustainability. In: Environmental Politics 26, 2, 193-211. https://doi.org/ 10.1080/09644016.2016.1256961

Purcell, M. (2009): Resisting neoliberalization: Communicative planning or counter-hegemonic movements? In: Planning Theory 8, 2, 140-165. https://doi.org/10.1177/ 1473095209102232

Reusswig, F.; Lass, W.; Bock, S. (2020): Abschied vom NIMBY. Transformationen des Energiewende-Protests und populistischer Diskurs. In: Forschungsjournal Soziale Bewegungen 33, 1, 140-160. https://doi.org/10.1515/ fjsb-2020-0012

Sack, D. (2019): Partizipation. In: Comtesse, D.; FlügelMartinsen, O.; Martinsen, F.; Nonhoff, M. (Hrsg.): Radikale Demokratietheorie. Ein Handbuch. Berlin. 671-680.

Sager, T. (2018): Communicative planning. In: Gunder, M.; Madanipour, A.; Watson, V. (Hrsg.): The Routledge Handbook of Planning Theory. New York, 93-104.

Sager, T. (2020): Populists and planners: 'We are the people. Who are you?' In: Planning Theory 19, 1, 80-103. https://doi.org/10.1177/1473095219864692

Sartori, G. (1997): Demokratietheorie. Darmstadt.

Schaal, G. S. (2012): Oligarchie als bessere Demokratie. In: Lembcke, O.; Ritzi, C.; Schaal, G. S. (Hrsg.): Zeitgenössische Demokratietheorie. Band 1: Normative Demokratietheorien. Wiesbaden, 445-472. https://doi.org/ 10.1007/978-3-531-94161-5_15

Schmidt, M. G. (2019): Demokratietheorien. Eine Einführung. Wiesbaden. https://doi.org/10.1007/978-3-65825839-9

Schumpeter, J.A. (2018 [1942]): Kapitalismus, Sozialismus und Demokratie. Stuttgart.

Selk, V.; Kemmerzell, J.; Radtke, J. (2019): In der Demokratiefalle? Probleme der Energiewende zwischen Expertokratie, partizipativer Governance und populistischer Reaktion. In: Radtke, J.; Canzler, W.; Schreurs, M. A.; Wurster, S. (Hrsg.): Energiewende in Zeiten des Populismus. Wiesbaden, 31-66. https://doi.org/10.1007/978-3658-26103-0_2

Swyngedouw, E. (2011): Depoliticized environments: The end of nature, climate change and the post-political condition. In: Royal Institute of Philosophy Supplement 69, 253-274. https://doi.org/10.1017/S1358246111000300

Trapenberg Frick, K. (2013): The actions of discontent: Tea Party and property rights activists pushing back against regional planning. In: Journal of the American Planning Association 79, 3, 190-200. https://doi.org/10. 1080/01944363.2013.885312

Weber, M. (1990): Wirtschaft und Gesellschaft. Tübingen. Wiechmann, T. (2019): Einleitung - Zum Stand der deutschsprachigen Planungstheorie. In: Wiechmann, T. (Hrsg.) 
: ARL Reader Planungstheorie. Band 1: Kommunikative Planung - Neoinstitutionalismus und Governance. Berlin, $1-11$.

Wirth, P.; Leibenath, M. (2017): Die Rolle der Regionalplanung im Umgang mit Windenergiekonflikten in Deutschland und Perspektiven für die raumbezogene Forschung. In: Raumforschung und Raumordnung | Spatial Resea rch and Planning 75, 4, 389-398. https://doi.org/10. 1007/s13147-016-0436-1

Zimmermann, K. (2019): Kommunikative Planung. In: Wiechmann, T. (Hrsg.): ARL Reader Planungstheorie. Band 1: Kommunikative Planung - Neoinstitutionalismus und Governance. Berlin, 14-23. 